
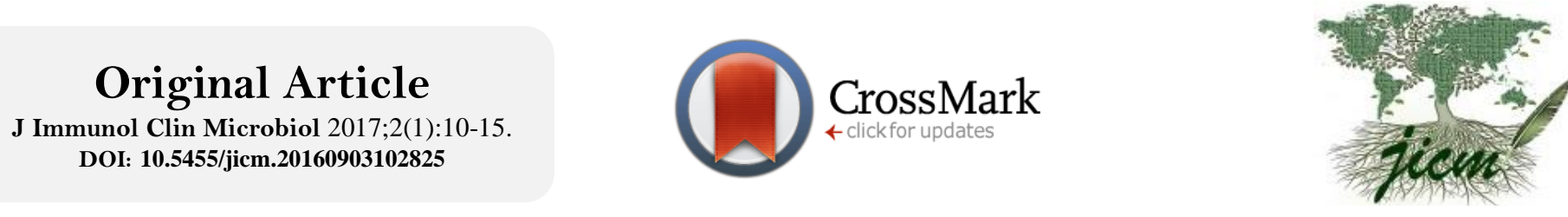

J Immunol Clin Microbiol

\title{
Sudden outbreak of Shigella among food handlers working at university student`s cafeteria
}

\author{
Birhanemeskel Tegene $^{1 *}$, Mulate Dagnew ${ }^{1}$, Feleke Moges ${ }^{1}$, Yetayah Wondemeneh ${ }^{2}$, Tesfaye Nega ${ }^{3}$ \\ ${ }^{1}$ Department of Medical Microbiology, Faculty of Medicine and Health Sciences, University of Gondar, Ethiopia. \\ ${ }^{2}$ Department of Medical Parasitology, Faculty of Medicine, University of Gondar, Ethiopia. \\ ${ }^{3}$ Training and Laboratory officer at Management Science at Health, Ethiopia.
}

\begin{abstract}
Background: Food borne disease outbreaks have resulted from unhygienic food handling and preparation practices within food establishments consequently have severe social and economic impacts on the human population. This study was aimed at exploring the prevalence of Shigella species and $S$. typhi carrier rate among food handlers at Gondar University student's cafeteria.

Material and Methods: A cross sectional survey was conducted among food handlers working at Gondar University students' cafeteria. A pre-tested structured questionnaire was used for collecting data. Stool samples were investigated for Shigella species outbreak and $S$. typhi as per the standard of laboratory methods.

Results: Among 395 food handlers, females comprised 289 (73.2\%). The majority 220 (55.7\%) were young adults 1828 years. Fifty three $(13.4 \%)$ of the food handlers have active diarrhea during the study period. Stool cultures revealed the isolation of $10(2.5 \%)$ Shigella species. No $S$. typhi was isolated. All shigella species were sensitive for ciprofloxacin and ceftriaxone. Thirty percent of the isolates were resistant for amoxicillin and tetracycline.

Conclusion: Isolation of Shigella species from food handlers pose significant risk on the student's population being served in the cafeteria. Therefore, it is essential for University of Gondar to implement food handlers training on food safety, conduct periodic medical checkup and continuous monitoring of personal hygiene.

Key words: Food handlers, Shigella species, Gondar
\end{abstract}

\section{Introduction}

Food safety remains a critical issue nowadays among professionals in the food service sector as well as consumers $(1,2)$. This is basically due to outbreaks of food-borne diseases resulting in substantial costs to individuals and the economy (3) and indeed the widespread and increasing incidence of food-borne diseases has severe social and economic impacts on the human population (4).

Reports of food borne disease outbreaks in various countries have resulted from unhygienic food handling and preparation practices within food establishments (5). In countries where disease surveillance is well established, food borne diseases are well monitored.

*Corresponding Author: Birhanemeskel Tegene, Department of Medical Microbiology, Faculty of medicine and health science, University of Gondar, Ethiopia E-mail: meskelbirehane@gmail.com Received: Jul 21, 2016 Accepted: Sep 03, 2016 Published Online: Feb 15, 2017.
There were about 737 outbreaks of food borne diseases with a total of 52,011 cases reported to the Centers for Disease Control and Prevention (CDC) (6). Thirty three percent of these outbreaks were related to food consumption in restaurants, cafeterias and delicatessens (7).

Report in the USA show that an estimate of 76 million foods related illnesses are seen annually (8), a total of 23,010 cases of dysentery have been reported in Turkey in 1997 (9). Though illnesses and outbreak estimates are available for developed countries, lack of effective surveillance systems hampers availability of similar estimates for developing countries (10).

This is an Open Access article distributed under the terms of the Creative Commons Attribution Non-Commercial License (http://creativecommons.org/licenses/bync/4.0/) which permits unrestricted non-commercial use, distribution, and reproduction in any medium, provided the original work is properly cited. 
However, the problem is severe in developing countries due to difficulties in securing optimal hygienic food handling practices. In developing countries, up to an estimated $70 \%$ of cases of diarrheal disease are associated with the consumption of contaminated food (11).

Food-handlers with poor personal hygiene working in food-serving establishments could be potential sources of infections of many enteropathogenic bacteria (12). Food handlers who harbour enteropathogenic bacteria may contaminate foods with their faeces via their fingers, which in turn lead to food processing, and finally to infection of consumers (13).

Shigellosis is one of enteric bacteria which is endemic in many developing countries and also occurs in epidemics causing considerable morbidity and mortality. Major obstacles to the control of shigellosis include the ease with which it spreads from person to person and the rapidity with which it develops antimicrobial resistance (14).

Annually, there are 165 million cases of shigellosis resulting in 1.1 million deaths in the developing world (15). Study done in Gondar town showed that Shigella species were isolated from stool sample of four foodhandlers (3.1\%) out of 127 food handlers (16).

However, published information about food borne pathogens among food handlers is scarce in Ethiopia. Therefore, this study was undertaken to assess prevalence of Shigella out break and Salmonella carrier among food handlers in Gondar university cafeteria, Gondar, Ethiopia.

\section{Methods and materials}

A laboratory based cross sectional study was conducted among food handlers working at University of Gondar students cafeterias from October 1 to 15, 2011. Gondar town is located in Northwest Ethiopia. It has a population density of 300,000 . During the study period, Gondar University has about 25,000 numbers of students in regular and extension programs.

The university has three cafeterias, namely College of Medicine Health Sciences (CMHS), Tewodros and Maraki. The cafeterias were served for 10,000 students. All food handlers working at University of Gondar students cafeterias during the study period were the study population. All food handlers were composed from the three campuses were included in the study, except those who had taken antibiotics within the three weeks prior to the study. A pretested structured questionnaire was used for collecting information on demographic characteristics and clinical status of each food handler. Stool specimen were collected from each food handler with leak proof stool cup by experienced laboratory technologist and enriched with Selniete F broth. Two $(2 \mathrm{ml})$ of venous blood was also collected by laboratory technologist from food handlers for Widal test.

All stool specimens were inoculated into Selenite-F broth (Oxoid UK) and incubated for 24 hours at $37 \mathrm{oC}$ followed by subculture on MacConkey (Oxoid) at 37oC for 24 hours for isolation of Shigella and Salmonella species. The plates were examined for growth of bacteria and Gram stain was done for identification of gram reaction. Biochemical tests were performed on pure colonies from primary cultures for final identification of the isolates. Gram-negative rods were identified by performing a series of biochemical tests (Oxoid Limited). Namely, triple sugar iron agar, indole, Simon's citrate agar, lysine iron agar, urea, mannitol and motility (17).

Susceptibility testing was performed on isolates using agar disc diffusion technique recommended by Bauer et al (18). ampicilin $(10 \mu \mathrm{g})$, tetracycline $(30 \mu \mathrm{g})$, chloramphenicol $(30 \mu \mathrm{g})$, gentamycin $(10 \mu \mathrm{g})$ and norfloxacillin $(10 \mu \mathrm{g})$ cotrimoxazole $(25 \mu \mathrm{g})$ and ciprofloxacin $(10 \mu \mathrm{g})$ for Gram negative isolates. The resistance and sensitivity were interpreted according to the Clinical and Laboratory Standards Institute (CLSI) (19).

Widal test was done using $\mathrm{S}$. typhi $\mathrm{O}$ and $\mathrm{H}$ antigens according to the manufacturer's instruction. In brief, the test was done by mixing one drop of serum with one drop each of $\mathrm{O}$ and $\mathrm{H}$ antigens separately on glass slide. After rocking the slide back and forth, the mixture was observed for macroscopic agglutination. If there was agglutination within one minute it was reported as positive, otherwise as negate. All materials, equipment and procedures were adequately controlled. Culture media was tested for sterility and performance. International control bacteria strains, E. coli (American Type Culture Collection [ATCC] 25922) and S. thipy (ATCC 25923) P. aeruginosa (ATCC 27853) were used in controlling the tests carried out.

Data were entered and analyzed using SPSS statistical analysis software version 20. Categorical datas are presented as absolute and relative frequencies using frequency counts as $\mathrm{n}(\%)$. whereas Continuous data were described as mean + standard deviation. The chisquare test was employed to assess the association between variables. A p-value of less than 0.05 was considered as statistical significance.

The data were collected after written informed consent obtained from all study participants, and the study was approved by the Research Ethics Committee of the University of Gondar. The confidentiality of the study subject result was kept at all times. The food handlers 
who were positive for Shigella and Salmonella were referred to the University of Gondar Hospital for treatment and treated as per guideline of the Hospital.

\section{Results}

\section{Sociodemographic Characteristics'}

A total of 395 food-handlers were included in the study. $289(73.2 \%)$ were females. Their mean age was 30.9 years, ranging from 18-68 years. The majority 220 $(55.7 \%)$ of the food-handlers were young adults aged 18 28 years. The sociodemographic characteristics of food handlers are shown in Table 1.

Table 1. Sociodemographic characteristics of food handlers working at Gondar university cafeteria in october1-15, 2011.

\begin{tabular}{lll}
\hline Characteristics & Number & Percent \% \\
\hline Age in years & & \\
\hline $18-28$ & 220 & 55.7 \\
$29-39$ & 93 & 23.5 \\
$40-50$ & 63 & 15.9 \\
$51-60$ & 15 & 3.8 \\
$61+$ & 4 & 1 \\
\hline Sex & & \\
\hline Male & 106 & 26.8 \\
Female & 289 & 73.2 \\
\hline
\end{tabular}

Fifty three (13.4\%) of the food handlers have active diarrhea during the study period only four individuals have had a history of vomiting and other clinical characteristics of food handlers is shown in (Table 2).

Table 2. Clinical characteristics of food handlers working at Gondar university cafeteria in October, 1-15, 2011. A total of 395 food handlers screened, stool cultures

\begin{tabular}{|c|c|c|}
\hline Characteristics & Number & Percent \% \\
\hline \multicolumn{3}{|c|}{ History of vomiting } \\
\hline Yes & 4 & 1.01 \\
\hline No & 391 & 98.99 \\
\hline \multicolumn{3}{|l|}{ Bloody stool } \\
\hline Yes & 7 & 1.8 \\
\hline No & 388 & 98.2 \\
\hline \multicolumn{3}{|l|}{ Abdominal pain } \\
\hline Yes & 53 & 13.4 \\
\hline No & 342 & 86.6 \\
\hline \multicolumn{3}{|c|}{ History of diarrhea } \\
\hline Yes & 53 & 13.4 \\
\hline No & 342 & 86.6 \\
\hline \multicolumn{3}{|l|}{ Fever } \\
\hline Yes & 27 & 6.8 \\
\hline No & 368 & 93.2 \\
\hline Total & 395 & 100 \\
\hline
\end{tabular}

revealed the isolation of 10(2.5\%) of Shigella species. However; salmonella species was not isolated in food handlers of stool culture. On the other hand, Widal test positive for $\mathrm{O}, \mathrm{H}$ and for both are $(33.7 \%),(2.3 \%)$, $(10.6 \%)$ respectively Widal test result for food handlers is shown in (Table 3).

Table 3. Widal test positive for food handlers working at Gondar university cafeteria in October, 1-15, 2011.

\begin{tabular}{clll}
\hline & & Type of test & \\
\hline Test result & $\begin{array}{l}\text { Somatic } \\
\text { antigen "O" }\end{array}$ & $\begin{array}{l}\text { Flagella } \\
\text { antigen "H" }\end{array}$ & $\begin{array}{l}\text { For both } \\
\text { antigen }\end{array}$ \\
Positive & $133(33.7 \%)$ & $9(2.3 \%)$ & $42(10.6 \%)$ \\
Negative & $262(66.3 \%)$ & $386(97.7 \%)$ & $353(89.4 \%)$ \\
\hline
\end{tabular}

\section{Antimicrobial susceptibility}

All shigella species were sensitive for ciprofloxacin and ceftriaxone. Thirty percent of the isolates were resistant for amoxicillin and tetracycline. The Antimicrobial susceptibility pattern of shigella species isolated from food handlers are shown in (Table 4).

Table 4. Antimicrobial susceptibility pattern of 10 shigella species isolated from food handlers at the University of Gondar students cafeterias from in october1-15, 2011.

\begin{tabular}{lcc}
\hline $\begin{array}{l}\text { Antimicrobial } \\
\text { agents }\end{array}$ & $\begin{array}{c}\text { Sensitivity } \\
\text { number } \\
\text { \% }\end{array}$ & $\begin{array}{c}\text { Resistance number } \\
(\%)\end{array}$ \\
\hline Ciprofloxacin & $10(100 \%)$ & $0(0 \%)$ \\
Chloramphenicol & $10(100 \%)$ & $0(0 \%)$ \\
Norfloxacillin & $9(90 \%)$ & $1(10 \%)$ \\
Ceftriaxone & $10(100 \%)$ & $0(0 \%)$ \\
Tétracycline & $7(70 \%)$ & $3(30 \%)$ \\
SXT & $8(80 \%)$ & $2(20 \%)$ \\
Amoxicillin & $7(70 \%)$ & $3(30 \%)$ \\
\hline
\end{tabular}

\section{Relation of variables with shigella positivity}

There was no statistical significant association between isolated shigella species and sex. (Chi-Square $=1.17, \mathrm{P}$ value $=0.73$ ). Those study participants having vomiting more likely to be positive for Shigella species when we compared with others clinical predictor. (Chi-Square $=$ $47.8, \mathrm{P}$ value $=0.003$ ). Even though it is not significant, those having diarrhea more likely to be positive for Shigella species $(\mathrm{OR}=4.56)$ and other clinical 
characteristics of food handlers relation is shown in (Table 5).

Table 5. Sociodemographic and clinical characteristics of food handlers in relation to shigella isolates from food handlers at Gondar students cafeterias from October 1-15, 2011.

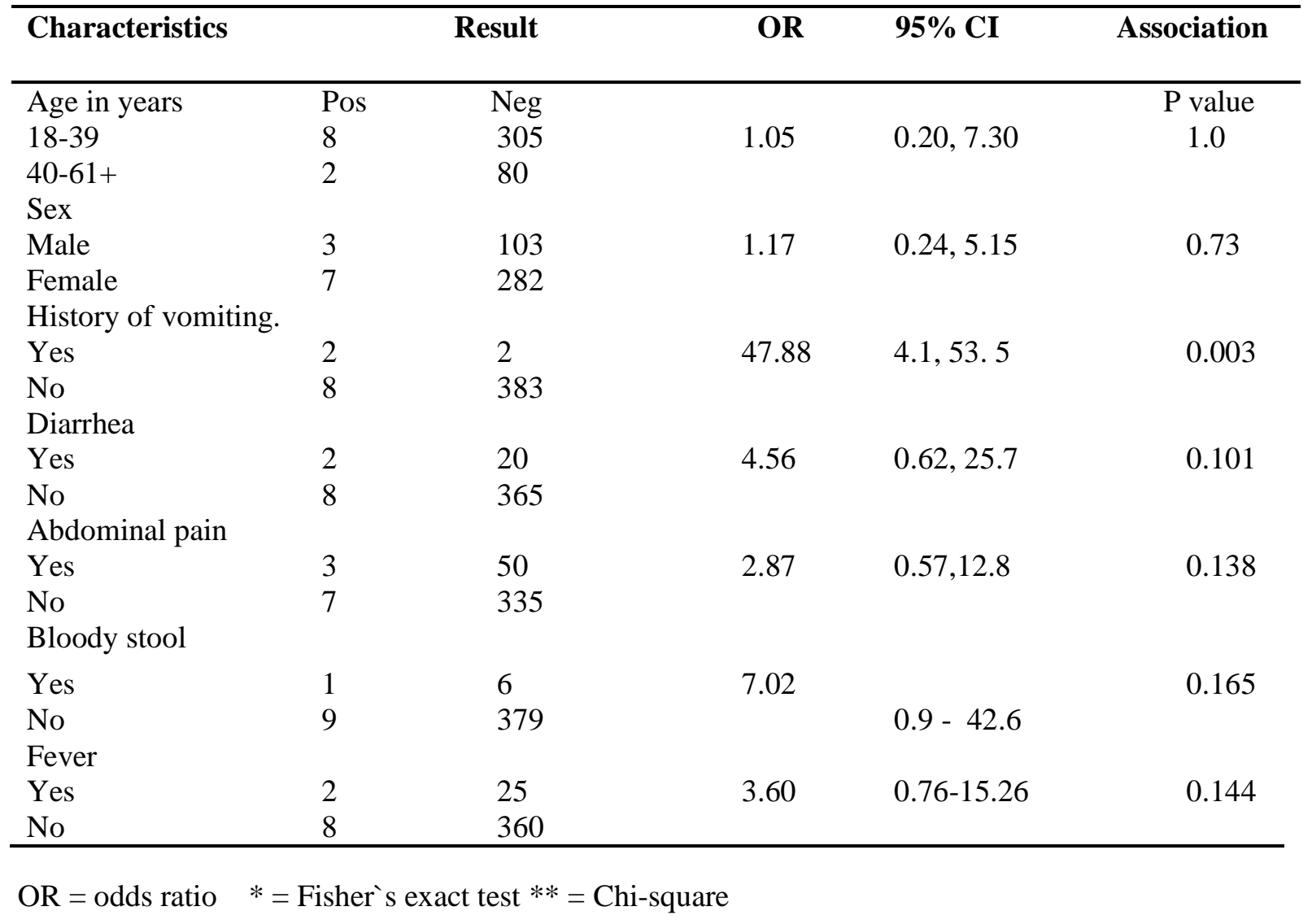

\section{Discussion}

Food safety remains a critical issue nowadays among professionals in the food service sector as well as consumers $(1,2)$. This is basically due to outbreaks of food-borne diseases resulting in substantial costs to individuals and the economy (3) and indeed the widespread and increasing incidence of food-borne diseases has severe social and economic impacts on the human population (4). Thus, the data presented in this study could provide information of immediate public health importance to clinicians in northwest Ethiopia on the selection of antimicrobial agents for the treatment of patients suffering from acute shigella dysentery. First there was shigella outbreak among students population. Students had sever vomiting and diarrhea. Their stool culture showed Shigella dysentery. The outbreak continues and it had seen among food handlers. The source of the outbreak was investigated and surveyed among food handlers.

The result showed that food handlers were positive for Shigella species. These indicate that the hygienic practice of the food handlers working in catering establishments of the study site is not satisfactory.
Prevalence rate of Shigella species in food handlers was $(2.5 \%)$. This indicates that positive individuals were suffering from bacillary dysentery right at that point in time: it is logical to suggest, therefore, that these food handlers might have transferred the Shigella infection to the students population they serve.

Our finding is in agreement with the study reported (3.1\%) from food handlers previously in Gondar (16). However, this finding was lower than the finding reported as $90(7.5 \%)$ in pediatric patients in the University of Gondar Hospital [20]. This may be due to the difference in technique, type of study participant and the sample size. The present study is higher than reports from Sudan (1.3\%) and Jordan (1.4\%) $(21,22)$.

It is important to note that Shigella organisms do not have any natural reservoirs in animals and spread only from person to person and outbreak are most often in facilities such as day care center, and cafeterias, and similar settings (23).

All Shigella isolates in our study were sensitive to ciprofloxacin and showed $10 \%$ resistance to norfloxacin (Table 2).This is more or less similar to study done by Andualem et al $100 \%$ and $90 \%$ sensitive to norfloxacin 
and ciprofloxacin (25). However, this finding is lower resistance when compared to a study reported in the University of Gondar Hospital [16] which shows high resistance to tetracycline (90\%), cotrimoxazole $(84.6 \%)$, ampicilin (78.9\%) and chloramphenicol (67.8\%) and lower resistance to gentamycin (12.2\%) (19). As opposed to the $62 \%$ resistant to chloramphenicol that has been reported in a study in Gondar(25),the frequency of shigella strain resistant to against chloramphenicol was $0 \%$. This is may be due to the former study is done in HIV /AIDS patients that were taking antibiotics frequently.

Salmonella species was not isolated from the stool cultures of food handlers in the present study. This is similar to study done in Gondar town previously (16). Although the sources of Salmonella species are intestinal tract of animals and humans, their carriage in food handlers was very rare [19]. However, a sudden outbreak of food poisoning due to salmonella was reported as $79(23 \%)$ ten years ago, in the students population of Gondar College of Medical Sciences (24).

On the other hand, Widal test positive for $\mathrm{O}, \mathrm{H}$ and for both $\mathrm{A}$ and $\mathrm{H}$ were $(33.7 \%),(2.3 \%),(10.6 \%)$ respectively. This finding is comparable to study done in Gondar, 42(14\%), 30(10\%), and 18(6\%) food handlers were positive $\mathrm{O}, \mathrm{H}$ and for both, respectively (26).

\section{Conclusion and Recommendations}

Isolation of Shigella species from food handlers pose significant risk on the students' population being served in the cafeteria. Therefore,

$\checkmark$ It is essential for University Hospital to implement food handlers training on food safety

$\checkmark$ Conduct periodic medical checkup and continuous monitoring of personal hygiene.

$\checkmark$ Emphasizes should be given to marak campus food handlers.

$\checkmark$ Efficient out-brake surveillance, including laboratory data, is necessary to monitor the disease trend, population at risk, serotype distribution and antimicrobial susceptibility in order to implement appropriate public health interventions against this out-brake

$\checkmark$ Laboratory equipments at least for routine purpose should be available at all campus laboratory

$\checkmark$ Further study should focus on the preventable aspect of outback

\section{Limitation}

The limitation of this outbreak investigation at the university cafeterias was a small number of reagents to carry out a salmonella and shigella serotyping which causing food borne diseases in Ethiopia in the study period.

\section{Acknowledgements}

We acknowledge University of Gondar Ethical review board. We greatly appreciate University of Gondar Hospital Laboratory for cooperation during the study. We are also grateful to the food handlers who participated in this study.

Ethics Committee Approval: Ethics Committee approval was received for this study from the ethics committee.

Informed Consent: N.A.

Peer-review: Externally peer-reviewed.

Conflict of Interest: No conflict of interest was declared by the author.

Financial Disclosure: The author declared that this study has received no financial support.

\section{References}

1. Scheule, B. Farm to Fork: Critical Control Points for Food Safety. Journal Nutrition in Recipe and Menu Development 2001, 3:3-27.

2. Badrie, N. Odwn R. Consumer Awareness and Perception to Food Safety Hazards in Trinidad, West Indies. Int $J$ Consumer Studies 2006, 17: 370-377.

3. Egan M.B, Raats M.M., Grubb S.M., Eves A., Lumbers M.L, Dean M.S. et al. A Review of Food Safety and Food Hygiene Training Studies in the Commercial Sector. Food Control, 2007, 18: 1180-1190.

4. Molins, R.A, Motarjemi, Y. and Käferstein F.K. Irradiation: a Critical Control Point in Ensuring the Microbiological Safety of Raw Foods. Food Control 2001, 12: 347-356.

5. Zeru K, Kumie A: Sanitary conditions of food establishments in Mekelle town, Tigray, North Ethiopia. Ethiop J Health Dev 2007, 21: 3-11.

6. Centers for Disease control and Prevention (CDC). Food borne Illness. International Food Safety Consultancy 2005.

7. Salvato Joseph A. Environmental Engineering and Sanitation. Fourth Edition. A Wiley Interscience publication, John Wiley \& Sons INC, New York, 1992.

8. Anding DJ, Boleman C, and Thompson B. Self-Reported Changes in Food Safety Behaviors among Food service Employees: Impact of a Retail Food Safety Education Program. Journal of Food Science Education/JFSE/ 2007, 6:7276.

9. Acikel CH, Ogur R, Yaren H, Gocgeldi E, Ucar M, Kir T. The Hygiene Training of Food Handlers at a Teaching Hospital. Food Control, 2007 19: 186-190.

10. Lynch M, Painter J, Woodruff R, Braden C, Surveillance for Food bore Disease Outbreaks, United States, 2002. MMWR 
Surveill Summ 2006, 55: 1-42.

11. Malhotra R., Lal P., Prakash K., Daga M., Kishore J. Evaluation of a Health Education Intervention on Knowledge and Attitudes of Food Handlers Working in a Medical Collage in Delhi, India. Asia Pac J Public Health, 2008 20: 277-286.

12. World Health Organization. Health surveillance and management procedures of food-handling personnel. Geneva: World Health Organization, 1999: 7-36. (Technical report series no. 785).

13. Kaferstein F, Abdussalam M: Food safety in the 21st century. Bull World Health Organ. 1999, 77: 347-51.

14. World health Orgainzation. Guidelines for the control of shigellosis, including epidemics due to Shigella dysenteriae type 1 .WHO2005.

15. Michael E, Mohammad A, Mohammad Y. Risk areas and neighborhood-level risk factors for Shigella dysenteriae 1 and Shigella flexneri. Healthplace 2008, 14: 96-105.

16. Andargie G, Kassu A, Moges F, Tiruneh M, Huruy K (2008) Prevalence of bacteria and intestinal parasites among food-handlers in Gondar Town, Northwest Ethiopia. $J$ health popul Nutr 26(4), 445-451.

17. WHO: Basic laboratory Procedures in Clinical Bacteriology. 2nd edition. Geneva: WHO; 2003. P.37-50.

18. Bauer AW, Kirby WM, Sherris JC, Turch M: Antibiotic susceptibility testing by standard single disk method. Am J Clin Pathol 1966, 45: 493-496.

19. Clinical and Laboratory Standards Institute. Methods for determining bactericidal activity of antimicrobial agents. Tentative Guidelines, M26-TCLSI. Villanova, PA; 1993.

20. Tiruneh M. Serodiversity and antimicrobial resistance pattern of Shigella isolates at University of Gondar teaching Hospital, Northwest Ethiopia. Japan J Infect Dis 2009 62: 9397.

21. Ahmed., Hassan H., Bacteriological and parasitological assessment of food handlers in the Omdurman area of Sudan. $J$ Microbiol Immunol Infect 2010. 43,70-73.

22. Al-Laham AB, Abu-Saud M, Shehabi AA. Prevalence of Salmonella, Shigella and intestinal parasites in food handlers in Irbid, Jordan. J Diarrheal Dis Res 1990, 8, 160-162.

23. Tortora GJ. Microbiology an introduction.9th ed. India: Kidersly 2009.

24. Assefa A, Mengistu G, Tiruneh M Salmonella Newport: outbreak of food poising among college students due to contaminated undercooked eggs, Northwest Ethiopia. Ethiop Med J 1994 32: 1-5.

25. Andualem B, Kassu A, Diro E, Moges F, Gedefaw M. The prevalence and antimicrobial responses of Shigella isolates in HIV-1 infected and uninfected adult diarrhoea patients in north west Ethiopia. Ethiop J Health Dev 2006; 20: 99-105.

26. Dagnew M, Tiruneh M, Moges F, Gizachew M. Bacterial Profile and Antimicrobial Susceptibility Pattern among Food Handlers at Gondar University Cafeteria, Northwest Ethiopia. J Infect Dis Ther (2013) 1:105. doi:10.4172/2332-0877.1000105

\section{How toite?}

Tegene B, Dagnew M, Moges F, Wondemeneh Y, Nega T. Sudden outbreak of Shigella among food handlers working at university student`s cafeteria. J Immunol Clin Microbiol 2017; 2(1):10-15.

DOI: http://dx.doi.org/10.5455/jicm.20160903102825

Submit your next manuscript to the JICM and take full advantage of:

- Convenient online submission,

- Thorough peer review, Fast Response,

- No charges,

- Immediate publication on acceptance,

- Inclusion in Scopemed and High quality indexes,

- Research which is freely available for redistribution of the worldwide literature

To submit your manuscript, click on http://www.jiacm.com

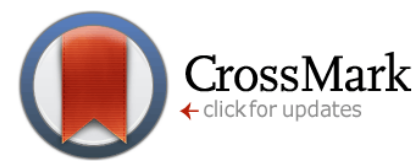

Published by The QMEL.org

International Medical Education Library

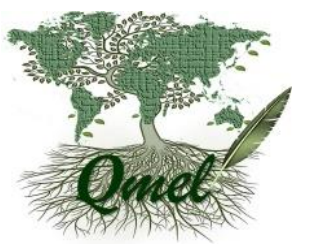

